\title{
Sympatric speciation in the genomic era
}

Foote, Andrew

\section{Trends in Ecology and Evolution}

\author{
DOI: \\ 10.1016/j.tree.2017.11.003
}

Published: 01/02/2018

Cyswllt i'r cyhoeddiad / Link to publication

Dyfyniad o'r fersiwn a gyhoeddwyd / Citation for published version (APA):

Foote, A. (2018). Sympatric speciation in the genomic era. Trends in Ecology and Evolution, 33(2), 85-95. https://doi.org/10.1016/j.tree.2017.11.003

\footnotetext{
Hawliau Cyffredinol / General rights

Copyright and moral rights for the publications made accessible in the public portal are retained by the authors and/or other copyright owners and it is a condition of accessing publications that users recognise and abide by the legal requirements associated with these rights.

- Users may download and print one copy of any publication from the public portal for the purpose of private study or research.

- You may not further distribute the material or use it for any profit-making activity or commercial gain

- You may freely distribute the URL identifying the publication in the public portal ?
}

Take down policy

If you believe that this document breaches copyright please contact us providing details, and we will remove access to the work immediately and investigate your claim. 


\section{Opinion Submission for Trends in Ecology and Evolution}

\section{Sympatric Speciation in the Genomic Era}

\section{Andrew D. Foote}

3 Molecular Ecology and Fisheries Genetics Laboratory, School of Biological Sciences, Bangor University,

Bangor, Gwynedd, LL57 2UW, UK

6 Keywords: sympatric speciation; genomics; divergence-with-gene-flow

7

8 Sympatric speciation has been of key interest to biologists investigating how natural and

9 sexual selection drive speciation without the confounding variable of geographic isolation.

10 The advent of the genomic era has provided a more nuanced and quantitative

11 understanding of the different and often complex modes of speciation by which sympatric sister taxa arose, and a re-assessment of some of the most compelling empirical case studies of sympatric speciation. However, I argue that genomic studies based on contemporary populations may never be able to provide unequivocal evidence of true primary sympatric speciation, and there is a need to incorporate palaeogenomic studies in to this field. This inability to robustly distinguish cases of primary and secondary divergence-with-gene-flow may be inconsequential, as both are useful for understanding the role of large effect barrier loci in the progression from localised genic isolation to genome-wide reproductive isolation. I argue that they can be of equivalent interest due to shared underlying mechanisms driving divergence and potentially leaving similar coalescent patterns. 


\section{Opinion Submission for Trends in Ecology and Evolution}

\section{A Century of Contention Over Sympatric Speciation}

Primary sympatric speciation is the evolution of reproductive isolation without geographic barriers, in which new species arise from a single ancestral population [1-5]. As these criteria do not allow for any physical separation between the incipient species, the potential for interbreeding and gene flow remains throughout the speciation process, from inception to completion. Recombination can therefore break up linkage between alleles beneficially associated with environmental variation, and alleles associated with incompatibilities and reproductive isolation [6]. As such, it is the most extreme, restrictive and arguably the most controversial scenario of divergence-with-gene-flow [7-11]. Thus, the existence and relevance of this mode of speciation in nature has been hotly debated for over a century [1-11]. The continued great interest for evolutionary biologists in sympatric speciation is understanding the seemingly rare conditions and processes under which natural and sexual selection can drive ecological divergence and reproductive isolation in a continuously distributed population $[4,7]$, as compared with allopatric speciation, in which geographic barriers initiate reproductive isolation and population divergence follows [2-8]. Under the latter scenario, it can be difficult to establish the extent of the role of selection due to ecological variation relative to intrinsic barriers developed during geographic isolation in promoting reproductive isolation [12].

After over a century of debate, and despite its theoretical plausibility and some apparently compelling empirical examples, many facets of sympatric speciation remain controversial. Given this, a recent review on speciation argued that the debate over allopatric versus sympatric speciation was unproductive and should not be a significant part of the future research agenda [13]. However, as per the oft-quoted prediction by Mayr: 'Sympatric speciation is like the Lernaean hydra which grew two new heads whenever one of its old heads was cut off.....the issue will be raised again at regular intervals' [8]. The advent of high-throughput sequencing, coupled with the development and application of population genomic methods that allow the inference of complex evolutionary histories, have led to a resurgent interest in sympatric speciation and a re-assessment of some of the most compelling empirical case studies $[14,15,16]$.

In the genomic era, we can now quantify the genetic contribution of one or more ancestral populations to contemporaneously sampled sympatric daughter species. These advances have led to some of the most compelling examples of primary sympatric speciation being reconsidered as a product of multiple colonisations and secondary contact. Other examples 


\section{Opinion Submission for Trends in Ecology and Evolution}

appear to be robust. However, here I argue that such backward-in-time approaches have limited ability to distinguish between periods of spatial overlap, but the absence of gene flow (i.e., when no coalescence events take place between the ancestral incipient species), and the absence of gene flow during periods of spatial separation. I propose that a forward-in-time approach, utilising palaeogenomics may be a complementary approach that could leverage additional information in some contexts. Lastly, I consider whether primary and secondary sympatric speciation represent a mechanistic dichotomy, I suggest that primary and secondary contact can leave a similar genomic signature, when speciation is driven by tightly clustered or large effect loci. Arguably, the advent of affordable population genomic studies should place less focus on whether study systems result from primary or secondary contact and instead focus Thereby, facilitating on the mechanistic aspects of the genomic architecture and making progress in identifying the conditions and processes under which natural and sexual selection can drive speciation, without extrinsic barriers to gene flow [13].

\section{Genomic insights into the ancestral context of sympatric speciation}

A compelling empirical case study of primary sympatric speciation requires the robust inference of past biogeography; specifically, that the present day sympatric daughter species arose from a common ancestral population, with no period of geographic isolation (see Box 1). Prior to the genomic era, empiricists used phylogenetics and assumed that the geographic distribution of the ancestral population was the same as the present-day daughter species, if they formed monophyletic species pairs or flocks in geographically isolated 'island' habitats [17-24]. However, a major limitation of the inference of sympatric speciation from the monophyletic relationship among sympatric species is that monophyly may result from several processes, other than true sympatric speciation (Figure 1). Modelling speciation as a bifurcating tree presents a point estimate of this evolutionary process [14] and does not consider the possibility that species derived ancestry from multiple source populations [25-27]. This is a key flaw with the criteria of Coyne and Orr [4]; monophyly of sympatric sister species is consistent with, but not exclusive to a scenario of sympatric speciation. It does not provide conclusive evidence that present day sympatric sister species emerged from a single colonisation, nor does it reject the alternative scenario of multiple colonisations in which monophyly results from introgression upon secondary contact [7,28]. 


\section{Opinion Submission for Trends in Ecology and Evolution}

90 However, these different scenarios do typically generate different patterns of genome-wide ancestry that can be used to distinguish between them. Under a scenario of sympatric speciation from a single source population, the daughter species will share a common ancestry, with segregating alleles being mainly those that are recently derived or were at low frequency in the ancestral population $[14,15,16]$. Alternatively, if sympatric sister species are the result of multiple colonisations and gene flow upon secondary contact, then each species should share differing proportions of ancestry with source outgroups (Figure 1). We can consider this as a continuum, from a single panmictic colonising population (Figure 1A); to colonisation by a hybrid swarm (Figure 1B); and lastly multiple colonisations and secondary contact following periods of geographic isolation (Figure 1C). This is a representative, but not an exhaustive list of possible scenarios that could generate the same consensus phylogentic pattern as sympatric speciation. Recently developed genomics methods can provide robust evidence of admixture and estimate ancestry proportions, even if gene flow events occurred hundreds of generations ago and under scenarios of incomplete lineage sorting and demographic change [29-32]. For example, the closely related $D$-statistic (ABBA-BABA) and $f$-statistic tests identify taxa that share an excess of ancestry (measured as derived alleles and allele frequencies respectively) with an outgroup [29,30]. The tract length of genomic regions inferred to have introgressed during secondary contact can provide further information on the timing of gene flow, and whether introgression pre- or post-dated sympatric diversification [33,34].

The application of such a population genomics approach has reassessed the sympatric origins of arguably some of the most compelling empirical examples of sympatric speciation: monophyletic species pairs and flocks of cichlids found in small uniform crater lakes in

113 Cameroon, Nicaragua and Tanzania $[14,15,16]$. The lakes were argued to be sufficiently small-

114 in-size; ecologically monotonous with no microgeographical barriers; and isolated from 115 outside riverine populations by the crater rim, that sympatric speciation appeared to be the most

116 likely biogeographical scenario under which these sister species had diverged $[17,18]$. In each 117 case, cichlid species within the lakes have diverged in ecologically-associated morphological 118 traits, and show evidence of reproductive isolation and monophyly, consistent with sympatric 119 origins [15,17-21,35]. However, analyses of genome-wide ancestry have revealed varying complexity in the evolutionary history of cichlids within each study area. These range from genomic ancestries that are best explained by multiple colonisations of Cameroon crater lakes and secondary gene flow following periods of allopatry [14]; to divergence in sympatry in

123 Nicaraguan crater lakes, but following secondary colonisation events and admixture prior to 


\section{Opinion Submission for Trends in Ecology and Evolution}

124 the radiations within each lake [16]; to what appears to be speciation following a single colonisation in a Tanzanian crater lake, albeit with some gene flow from the lake to nearby outgroup populations [15].

These descriptive results can then be developed into demographic models, allowing the estimation of ancestral divergence times, effective population sizes and migration rates, and the testing of alternative evolutionary scenarios (e.g. $[15,36,37])$. However, modelling whether sympatric populations diverged with gene flow, or whether migration took place sometime after the populations had diverged, consistent with secondary contact, requires the estimation of the timing and the number of migration events [38-40]. These parameters can be intractable, as genomic data from present day populations can be consistent with many migration and admixture scenarios, which result in the same coalescent times [39,40]. More general caveats also apply, for example, most models are oversimplified representations of biological reality, and only inputted models are tested. Model-based approaches are therefore best accompanied with model-free methods to identify a range of estimates for parameters, and scenarios to test. Additionally, there is a need to exclude non-neutral loci and account for genome-wide variation in effective migration and recombination [37,41].

142 The biological realism and relevance of the classification of the mode of speciation into the

143 discrete geographic categories such as sympatric, parapatric and allopatric has been questioned. Almost all candidate case studies of sympatric speciation have some degree of spatio-temporal differentiation between sister taxa, for example due to the patchy distribution of preferred habitat [14,42-44]. To countenance this, some have suggested that the relationship between taxa during the speciation process may be better quantified in a population genetics framework that quantifies key parameters such as migration rate [42]. This approach, and modelling sympatric speciation in general, relies on assuming a starting point of panmixia in

150 the ancestral population [5]. Yet this assumption of ancestral panmixia has been difficult or impossible to prove or reject in empirical case studies prior to the genomic era [42]. Others have argued for retaining a spatial component of sympatric speciation, in accordance with Mayr's definition [8]: that speciating sister taxa should be in 'cruising range' of each other throughout the speciation process [44]. However, in each case, the geographic context of speciation is divided into artificially discrete categories, whether they be based on spatial or genetic measures of separation [11]. Instead, the geographic context of speciation is perhaps 


\section{Opinion Submission for Trends in Ecology and Evolution}

158 the contribution of the shared ancestral population and any other contributing outgroup populations to the ancestry of the daughter species. Thereby providing a continuous and quantitative measure of the context and mode of speciation. This still does not fully resolve the uncertainty in the geographic context of divergence. For example, even among sympatric taxa with no detectable contribution from ancestral outgroups, as in Figure 1A, there may have been periods of spatial segregation among currently sympatric sister taxa. Ultimately, our ability to reconstruct the evolutionary history of sympatric sister taxa back to the shared ancestral population using backward-in-time genomic approaches, is constrained to being able to identify periods of gene flow through coalescent events, but is not able to distinguish periods of spatial overlap without gene flow from periods of spatial isolation.

Due to the timescales over which evolutionary processes such as adaptation and speciation take place, forward-in-time approaches are rarely utilised due to the limitations on the number of generations that can be sampled. However, the advent of palaeogenomics is expanding the scope of timescales over which we can sample genomes and look at genetic change from an ancestral population going forward in time to daughter species, and can complement hindcasting from contemporaneously sampled genomes. For example, sediment cores from post-glacial lakes can be used to sample lineages from the time the glaciers retreated to the present day (Figure 2). Such an approach has recently been applied to extract DNA from sediment of two lakes in Sweden, spanning the past 10,000 years, to reconstruct the colonisation and connectivity between whitefish (Coregonus lavaretus) ecotypes [45]. Whilst only very low concentrations of DNA are found in sediments, the sequencing of hard parts within the different layers of the sediment core, for example bones or spines, can yield genomic sequences that allow the tracking of genomic changes at QTL forwards in time.

\section{The genomic architecture of sympatric speciation}

184 The genomic architecture of a trait can be summarised as the number of underlying loci, their effect size and additivity, and their physical spacing across the genome. In addition to being shaped by recent and ongoing selection, this genomic architecture can be influenced by processes that include demographic history, linked selection in the ancestral population, recent and ongoing selection, and recombination rate [46].

Key questions in the study of sympatric speciation are how a genomic architecture shaped by gradual, incremental changes that occur under natural selection can account for rapid bursts of 


\section{Opinion Submission for Trends in Ecology and Evolution}

192 adaptive divergence; how localised genomic changes result in genome-wide reproductive 193 isolation; and how they can overcome the homogenising effect of ongoing gene flow [47-49].

194 Over the past decade genomic studies of adaptation have progressed from investigating single 195 or a few candidate genes to genome-wide studies, and have highlighted how divergence linked

196 to adaption can be widespread across the genome. Yet the chronology of genic change during 197 speciation, and how this progresses from individual 'barrier loci', through to genome-wide 198 differentiation (and how to study these processes), is still contentious and widely debated (see reference [49] and associated commentaries).

One of the primary approaches to exploring these questions has been to compare genome-wide variation in differentiation $\left(F_{\mathrm{ST}}\right)$ of allele frequencies across the 'speciation continuum'; i.e., between multiple pairs of sympatric and allopatric sister taxa that are at different stages of divergence $[47,48]$. This approach has been applied to multiple taxa, with varied results. While, most such studies to date have shown a progressive increase in the build-up of mean genomewide differentiation across the speciation continuum [50-53], and some have highlighted important barrier loci that reduce localised effective migration within some genomic regions due to being associated with adaptation and/or reproductive isolation [54,55]; many of these studies have identified alternative underlying causes of heterogeneity in the landscape of genomic differentiation [50-52]. These include reduced diversity from linked selection in the ancestral population, for example due to background selection (BGS) removing deleterious variants [56]; BGS is in-turn associated with variation in recombination rate and gene density in regions such as centromeres [57,58]; and selection on genome-wide smaller effect loci underlying polygenic traits. The genomic background of these different processes can then mask any potential signal from barrier loci associated with adaptation or reproductive isolation.

216 However, young examples of sympatric speciation may generate rare exemplar study systems,

217 in which there are clear 'genomic islands' which contain barrier loci associated with reproductive isolation and ecological diversification.

The effect size of a locus on a phenotypic trait has a positive correlative relationship with pleiotropy and deleterious effects [59], therefore adaptation is predicted to typically progress due to small changes in frequency across many alleles, each with a small additive phenotypic effect [60]. However, as noted above, in scenarios of ongoing gene flow during sympatry, recombination would be expected to break up linkage between loci associated with ecological adaptation and those associated with mate preference, thus counteracting ecologically driven 


\section{Opinion Submission for Trends in Ecology and Evolution}

226

227

228

229

230

231

232

233

234

235

236

237

238

239

240

241

242

243

244

245

246

247

248

249

250

251

252

253

254

255

256

257

258

259

speciation [6-9]. Additionally, the strength of selection on a locus is not just a function of its effect size and its interaction with the environment, it is also a function of effective population size $(\mathrm{Ne})$. The more robust examples of primary sympatric speciation are typically those that have colonised a remote, or closed, ecosystem prior to diverging, e.g. Lord Howe Island flora $[23,24]$ and crater lake cichlids [17-19]. Thus, it seems realistic that only a small number of initial colonisers founded these island or closed ecosystems. This founder effect is expected to greatly lower selection coefficients at loci of small effect that act additively on traits. Therefore, traits associated with ecological variation or mate choice that diverge during sympatric speciation, are more likely to be determined by loci tightly linked to each other in genomic regions of low recombination such as inversions [46,61], or be synergistically pleiotropic, i.e. so-called 'magic traits', which have a role in both ecological adaptation and assortative mating [62]. Therefore, these study systems are those that we expect barrier loci of large effect to be differentiated against a homogenous genome-wide background.

Recent genomic studies investigating quantitative trait loci (QTL) in model systems for speciation-with-gene-flow, have largely validated these predictions. For example, in Midas cichlids in Nicaraguan crater lakes, the highest effect size QTL for body shape and pharyngeal jaw morphology, both traits which show ecological-associated variation [20,21] are tightly clustered on a single chromosome and allele frequencies at these loci segregate in sympatric sister species [63]. Comparison of the genomes of benthic and littoral ecomorphs of Astatotilapia cichlids from a Tanzanian crater lake found regions of high differentiation and high divergence clustered mainly in five linkage groups harbouring genes associated with morphology and optical sensitivity, and therefore ecological variation and mate choice [15]. A recent study on sympatric populations of monkey flower species Mimulus laciatus and M. guttatus found that a few large effect size QTL explained much of the variance in flowering time and flower size traits [64]. Differences in flowering time are thought to be locally adaptive: $M$. laciatus, is found on dry exposed rocky outcrops and flowers earlier than $M$. guttatus to avoid the seasonal drought; and act as a prezygotic barrier to gene flow, therefore qualifying as a 'magic trait' [64]. Allochrony also plays a role in reproductive isolation between sympatric hawthorn and apple-infesting host races of the Rhagoletis pomonella fly, which differ in the intensity and timing of diapause [65]. SNP loci associated with the timing of diapause onset and diapause intensity were in several tightly linked clusters, thought to be within inversions [66]. 


\section{Opinion Submission for Trends in Ecology and Evolution}

260 The findings of these empirical studies are highly concordant with the predictions of most theoretical models of sympatric speciation, which require linkage between loci associated with reproductive isolation and loci associated with ecological adaptation, or pleiotropy in which ecological adaptation and reproductive isolation evolve simultaneously [67-69]. This contrasts with empirical examples in which a period of allopatry was important in segregating alleles associated with ecological variation. In examples of the latter scenario, intrinsic barriers can build up in many widespread genomic regions without recombination breaking them up during this allopatric phase. Thus, in many examples of sympatric speciation we anticipate large changes in allele frequencies at single or a few loci, while the rest of the genome is homogenised, until complete genome-wide isolation is established. Therefore, the coalescent times of the barrier loci are expected to pre-date the genome-wide time-to-most-recentcommon-ancestor (TMRCA) [70] (Figure 3). In contrast, if genome-wide polygenic adaptation and reproductive incompatibilities have evolved in allopatry, prior to secondary contact, then the TMRCA of the loci associated with reproductive isolation will be within the genome-wide range and need not be associated with large changes in allele frequencies, making them cryptic to genome-wide scan methods.

Strict primary divergence-with-gene-flow may not be needed for studying the evolution of large effect barrier loci against a homogenous genomic background. In theory, this pattern, could also be expected even if the genetic underpinning of divergent ecological adaptation and reproductive isolation develops during allopatry, and then segregates again after an initial period of mixing upon secondary contact, provided there is genome-wide homogenisation upon secondary contact (Figure 3). An allopatric phase and/or introgression events can facilitate speciation by intensifying disruptive selection and introducing new genomic variation that can act as a substrate for segregating polymorphisms under natural and sexual selection. Guerrero \& Hahn [71] recently suggested that balanced polymorphisms in the ancestral population, could sort upon splitting into daughter species, either due to ecological variation selecting for alternate alleles, or through selectively neutral sorting. They highlighted that such a process could explain the high absolute genetic divergence ( $\mathrm{D}_{\mathrm{XY}}$ ), suggestive of an ancient divergence, in the few genomic islands found when comparing the littoral and benthic ecomorphs of the Tanzanian crater lake Massoko. The two ecomorphs are estimated to have diverged only 5001,000 years, having diverged from the putative source population 10,000 years ago in a crater lake that formed $\sim 50,000$ years ago [15]. Guerrero \& Hahn [71] highlight that these regions containing putative balanced polymorphisms would form 'genomic islands' even without 


\section{Opinion Submission for Trends in Ecology and Evolution}

294 background $\mathrm{F}_{\mathrm{ST}}$ and $\mathrm{D}_{\mathrm{XY}}$ being lowered due to genome-wide homogenisation from gene flow.

295 However, it is not hard to imagine that these two forms could have arisen and collapsed

296 multiple times since colonising the crater, for example, due to episodic changes in water depth.

297 If negative frequency dependent selection maintained ecologically adaptive polymorphisms

298 even when the two forms collapse into an otherwise homogenous population, such a process

299 of repeated collapse and vicariance could mask any genomic signature of divergent origins in

300 the present-day populations, with the exception of balanced polymorphisms, which would coalesce much further back in time than the genome-wide mean TMRCA (Figure 3).

Lineage sorting and high genomic differentiation are also found at loci of large effect in the partially sympatric benthic-limnetic species pairs of threespine sticklebacks found in several lakes in British Columbia, Canada and hypothesised to have originated from a secondary invasion [72]. A PCA analysis of genome-wide neutrally evolving SNPs found a pattern of clustering by lake [73], which would be consistent with independent divergence of the benthic and limnetic forms of stickleback within each lake. However, SNPs evolving under natural selection grouped individuals by ecological niche, with further clustering of the older benthic form with geographically proximate single-form freshwater populations, whilst the younger limnetic form clustered more closely with marine populations [73]. These results are consistent with re-use of standing genetic variation from a second marine-to-freshwater colonisation, which then provided the raw genetic material for divergence within each lake driven by disruptive selection. Thus, the adaptation and speciation loci coalesce much further back in time, than the mean TMRCA of unlinked neutral loci. A further example is the sympatric hawthorn and apple-infesting races of Rhagoletis pomonella fruit fly, in which the inversion polymorphism influencing diapause traits evolved during an allopatric phase greater than a million years ago [74].

\section{Concluding remarks}

In the genomic era, sympatric speciation continues to be a controversial and much-debated 


\section{Opinion Submission for Trends in Ecology and Evolution}

\section{instances}

326 populations; for example, identifying where sympatric sister taxa that were thought to represent originating from a common ancestral population

327 a monophyletic group, are instead derived from multiple ancestral source populations [14].

328 However, there remains a bias towards being able to disprove primary sympatric speciation,

329 whilst generating conclusive evidence in support of primary sympatric speciation based on

330 hindcasting using modern genomes remain elusive. I suggest that palaeogenomics may have a

331 complementary role to play in future studies; for example, the sequencing of DNA from sediment cores can identify the temporal patterns of spatial overlap between two speciating lineages, even in the absence of gene flow. Lastly, the great interest of biologists in sympatric UNDERSTANDING

334 speciation has been how two lineages can diverge and become reproductively isolated in the

335 absence of extrinsic barriers. In the genomic era, we can study this process at the genic level.

336 In this review, I have highlighted several characteristics of the genomic underpinning of sympatric speciation, and that these can be found in examples of primary and secondary sympatric speciation. I therefore contend that it is the investigation of the process of sympatric speciation, rather than a dogmatic search for true primary sympatric speciation that will be most valuable to our understanding of speciation and adaptation at the genomic level.

342 Acknowledgements

343 I would like to thank the editor, Paul Craze, and Jeff Feder and one anonymous reviewers for

344 their constructive feedback, and Alex Papadopulos for useful discussions on this topic which 345 greatly improved this manuscript. Financial support was provided by the Welsh Government 346 and Higher Education Funding Council for Wales through the Sêr Cymru National Research 347 Network for Low Carbon, Energy and Environment, and from the European Union's Horizon 3482020 research and innovation programme under the Marie Skłodowska-Curie grant agreement 349 No 663830. 


\section{Opinion Submission for Trends in Ecology and Evolution}

351

352

353

354

355

356

357

358

359

360

361

362

363

364

365

366

367

368

369

370

371

372

373

374

375

376

377

378

379

380

381

382

\section{References}

1. Poulton, E.B. (1904) What is a species? Trans. Entomol. Soc. Lond. 1903, 77-116

2. Jordan, D.S. (1905) The origin of species through isolation. Science 22, 545-62

3. Mayr, E. (1942). Systematics and Origin of Species, Columbia University Press

4. Coyne, J. and Orr, H. (2004) Speciation, Sinauer Associates

5. Gavrilets, S. (2003) Models of speciation: what have we learned in 40 years? Evolution 57, 2197-2215

6. Slatkin, M. (1987) Gene flow and the geographic structure of natural populations. Science 236, 787-792

7. Bolnick, D.I. and Fitzpatrick, B.M. (2007) Sympatric speciation: models and empirical evidence. Annu. Rev. Ecol. Evol. Syst. 38, 459-487

8. Mayr, E. (1963) Animal Species and Evolution, Belknap

9. Felsenstein, J. (1981) Skepticism towards Santa Rosalia, or why are there so few kinds of animals? Evolution 35, 124-138

10. Jiggins C.D. (2006) Sympatric Speciation: Why the Controversy? Curr. Biol. 16, R333-R334

11. Butlin, R.K. et al. (2008) Sympatric, parapatric or allopatric: the most important way to classify speciation? Philos. Trans. R. Soc. Lond. B Biol. Sci. 363, 2997-3007

12. Bierne, N. et al. (2011) The coupling hypothesis: why genome scans may fail to map local adaptation genes. Mol. Ecol. 20, 2044-2072

13. Marie Curie Speciation Network (2012) What do we need to know about speciation? Trends Ecol. Evol. 27, 27-39

14. Martin, C.H. et al. (2015). Complex histories of repeated gene flow in Cameroon crater lake cichlids cast doubt on one of the clearest examples of sympatric speciation. Evolution 69, 1406-1422

15. Malinsky, M. et al. (2015) Genomic islands of speciation separate cichlid ecomorphs in an East African crater lake. Science 350, 1493-1498

16. Kautt, A.F. et al. (2016) Multispecies outcomes of sympatric speciation after admixture with the source population in two radiations of Nicaraguan crater lake cichlids. PLoS Genet. 12: e1006157

17. Schliewen, U.K. et al. (1994) Sympatric speciation suggested by monophyly of crater lake cichlids. Nature 368, 629-632 


\section{Opinion Submission for Trends in Ecology and Evolution}

18. Schliewen, U.K. and Klee, B. (2004) Reticulate sympatric speciation in Cameroonian crater lake cichlids. Frontiers Zool. 1:5

19. Barluenga M. et al. (2006) Sympatric speciation in Nicaraguan crater lake cichlid fish. Nature 439, 719-723

20. Elmer, K.R. et al. (2010) Rapid sympatric ecological differentiation of crater lake cichlid fishes within historic times. BMC Biology 8:60

21. Elmer, K.R. et al. (2010) Local variation and parallel evolution: morphological and genetic diversity across a species complex of neotropical crater lake cichlid fishes. Phil. Trans. R. Soc. B 365, 1763-1782

22. Filchak, K.E. et al. (2000) Natural selection and sympatric divergence in the apple maggot Rhagoletis pomonella. Nature 407, 739-742

23. Savolainen, V. et al. (2006) Sympatric speciation in palms on an oceanic island. Nature 441, 213-213

24. Papadopulos, A.S.T. et al. (2011) Speciation with gene flow on Lord Howe Island. Proc. Natl. Acad. Sci. 108, 13188-13193

25. Cavalli-Sforza, L.L. (1973) Analytic review: some current problems of human population genetics. Am. J. Hum. Genet. 25, 82-104

26. Cavalli-Sforza, L.L. and Piazza, A. (1975) Analysis of evolution: evolutionary rates, independence and treeness. Theor. Popul.Biol. 8, 127-165

27. Felsenstein, J. (1982) How can we infer geography and history from gene frequencies? J Theor. Biol. 96, 9-20

28. Schliewen, U.K. et al. (2006) Evolutionary biology—Evidence for sympatric speciation? Nature 444, E12-E13

29. Green, R.E. et al. (2010) A draft sequence of the Neandertal genome. Science 328, $710-722$

30. Durand, E.Y. et al. (2011) Testing for ancient admixture between closely related populations. Mol. Biol. Evol. 28, 2239-2252

31. Patterson, N. et al. (2012) Ancient admixture in human history. Genetics 192, 10651093

32. Peter, B.M. (2016) Admixture, population structure and F-statistics. Genetics 202, $1485-1501$

33. Harris, K. and Nielsen, R. (2013) Inferring demographic history from a spectrum of shared haplotype lengths. PLoS Genet 9:e1003521 


\section{Opinion Submission for Trends in Ecology and Evolution}

416

417

418

419

420

421

422

423

424

425

426

427

428

429

430

431

432

433

434

435

436

437

438

439

440

441

442

443

444

445

446

447

448

449

34. Lawson, D.J. et al. (2012) Inference of population structure using dense haplotype data. PLoS Genetics 8, e1002453

35. Schliewen, U.K. et al. (2001) Genetic and ecological divergence of a monophyletic cichlid species pair under fully sympatric conditions in Lake Ejagham, Cameroon. Mol. Ecol. 10, 1471-1488

36. Meier, J.I. et al. (2016) Demographic modelling with whole-genome data reveals parallel origin of similar Pundamilia cichlid species after hybridization. Mol. Ecol. doi: $10.1111 / \mathrm{mec} .13838$

37. Rougeaux, C. et al. (2017) Modeling the multiple facets of speciation-with-gene-flow towards inferring the divergence history of Lake Whitefish species pairs (Coregonus clupeaformis). Genome Biol. Evol. https://doi.org/10.1093/gbe/evx150

38. Strasburg, J. and Rieseberg, L. (2011) Interpreting the estimated timing of migration events between hybridizing species. Mol. Ecol. 20, 2353-2366

39. Sousa, V. C. et al. (2011) On the nonidentifiability of migration time estimates in isolation with migration models. Mol. Ecol. 20, 3956-3962

40. Juric, I. et al. (2016) The strength of selection against Neanderthal Introgression. PLoS Genetics 12: e1006340.

41. Sousa, V. C. et al. (2013) Identifying loci under selection against gene flow in isolation-with-migration models. Genetics 194, 211-233

42. Fitzpatrick, B.M. et al. (2008) What, if anything, is sympatric speciation? J. Evol. Biol. 21, 1452-1459

43. Babik, W. et al. (2009) How sympatric is speciation in the Howea palms of Lord Howe Island? Mol. Ecol. 18, 3629-3638

44. Mallet, J. et al. (2009) Space, sympatry and speciation. J. Evol. Biol. 22, 2332-2341

45. Olajos, F. et al. (2017) Estimating species colonization dates using DNA in lake sediment. Methods Ecol. Evol. DOI: 10.1111/2041-210X.12890

46. Lynch, M. and Walsh, B. (2007) The origins of genome architecture, Sinauer Associates

47. Feder, J.L. et al. (2012) The genomics of speciation-with-gene-flow. Trends in Genetics 28, 342-350

48. Seehausen, O. et al. (2014) Genomics and the origin of species. Nat. Rev. Genet. 15, 176-192

49. Ravinet, M. et al. (2017) Interpreting the genomic landscape of speciation: a road map for finding barriers to gene flow. J. Evol. Biol. 30, 1450-1477 


\section{Opinion Submission for Trends in Ecology and Evolution}

50. Renaut, S. et al. (2013) Genomic islands of divergence are not affected by geography of speciation in sunflowers. Nat. Comm. 4, 1827

51. Martin, S.H. et al. (2013) Genome-wide evidence for speciation with gene flow in Heliconius butterflies. Genome Res. 23, 1817-1828

52. Foote, A.D. et al. (2016). Genome-culture coevolution promotes rapid divergence of killer whale ecotypes. Nature Comm. 7, 11693

53. Vijay, N. et al. (2016) Evolution of heterogeneous genome differentiation across multiple contact zones in a crow species complex. Nat. Comm. 7, 13195

54. Nadeau, N.J. et al. (2012) Genomic islands of divergence in hybridizing Heliconius butterflies identified by large-scale targeted sequencing. Phil. Trans. R. Soc. B. 367 $343-353$

55. Jones, F.C. et al. (2012) The genomic basis of adaptive evolution in threespine sticklebacks. Nature 484, 55-61

56. Cruickshank, T.E. and Hahn, M.W. (2014) Reanalysis suggests that genomic islands of speciation are due to reduced diversity, not reduced gene flow. Mol. Ecol. 23, $3133-3157$

57. Ellegren, H. et al. (2012) The genomic landscape of species divergence in Ficedula flycatchers. Nature 491, 756-760

58. Burri, R. et al. (2015) Linked selection and recombination rate variation drive the evolution of the genomic landscape of differentiation across the speciation continuum of Ficedula flycatchers. Genome Res. 25, 1656-1665

59. Wagner, G.P. et al. (2008) Pleiotropic scaling of gene effects and the 'cost of complexity'. Nature 452, 470-472

60. Fisher, R.A. (1930) The Genetical Theory of Natural Selection. Oxford University Press

61. Yeaman, S. and Whitlock, M.C. (2011) The genetic architecture of adaptation under migration-selection balance. Evolution 65, 1897-1911

62. Servedio, M.R. et al. (2011) Magic traits in speciation: 'magic' but not rare? Trends Ecol.Evol. 26, 389-397

63. Fruciano, C. et al. (2016) Genetic linkage of distinct adaptive traits in sympatrically speciating crater lake cichlid fish. Nature Comm. 7:12736

64. Ferris, K.G. et al. (2017) The genetic architecture of local adaptation and reproductive isolation in sympatry within the Mimulus guttatus species complex. Mol. Ecol. 26, $208-224$ 


\section{Opinion Submission for Trends in Ecology and Evolution}

65. Bush, G.L. (1969) Sympatric host race formation and speciation in frugivorous flies of the genus Rhagoletis (Diptera Tephritidae). Evolution 23, 237-251

66. Ragland, G.J. et al. (2017) A test of genomic modularity among life-history adaptations promoting speciation with gene flow. Mol. Ecol. 26, 3926-3942

67. Dieckmann, U. and Doebeli, M. (1999) On the origin of species by sympatric speciation. Nature 400, 354-357

68. Fry, J.D. (2003) Multilocus models of sympatric speciation: Bush vs Rice vs Felsenstein. Evolution 57, 1735-1746

69. Gavrilets, S. (2004) Fitness Landscapes and the Origin of Species, Princeton University Press

70. Yang, M. et al. (2017) Can genomic data alone tell us whether speciation happened with gene flow? Mol. Ecol. 26, 2845-2849

71. Guerrero, R.F. and Hahn, M.W. (2017) Speciation as a sieve for ancestral polymorphism. Mol. Ecol. doi: 10.1111/mec.14290

72. Taylor, E.B. and McPhail, J.D. (2000) Historical contingency and ecological determinism interact to prime speciation in sticklebacks, Gasterosteus. Proc. R. Sci. B $267,2375-2384$

73. Jones, F.C. et al. (2012) A genome-wide SNP genotyping array reveals patterns of global and repeated species-pair divergence in sticklebacks. Curr. Biol. 83-90

74. Feder, J.L. et al. (2003) Allopatric genetic origins for sympatric host-plant shifts and race formation in Rhagoletis. Proc. Natl. Acad. Sci. 100, 10314-10319 


\section{Opinion Submission for Trends in Ecology and Evolution}

515 Barrier loci: genetic loci that cause reduced gene flow between speciating taxa at a localised

516 region of the genome.

517 Coalescent: when two lineages sampled from different populations merge back in time in a

518 commonly shared ancestral lineage.

519 Disruptive selection: selection that favours extreme phenotypes over intermediate

520 phenotypes within a population.

521 Divergence-with-gene-flow: the build-up of genetic and phenotypic differences, despite on-

522 going exchange of genes. This differentiation is typically driven by disruptive natural

523 selection. The term has been used inclusive of scenarios of divergence under ongoing gene

524 flow upon secondary contact, and is thus does not exclusively refer to sympatric speciation.

525 Ecomorph: a population which has distinctive ecological and morphological features.

526 Genomic islands: a region of the genome that is highly differentiated (estimated using $F_{\mathrm{ST}}$ )

527 between taxa compared with the genome-wide mean level of differentiation.

528 Magic trait: a trait subject to divergent selection and a trait contributing to mate choice

529 which are pleiotropic expressions of the same gene(s).

530 Monophyletic: belonging to a clade containing all the descendants of a single ancestor.

531 Panmixia: random mating within a population.

532 Parapatric speciation: the evolution of reproductive isolation in the absence of geographical

533 barriers to gene flow, in which the diverging populations have adjacent ranges.

534 Pleiotropic: an allele that has an effect on more than one trait.

535 Polymorphisms: genetic loci that have more than one allele.

536 Quantitative trait loci: genetic markers that are correlated with phenotype. These markers

537 contain, or are linked to, genes and regulatory regions associated with quantitative

538 phenotypic variation. 
Opinion Submission for Trends in Ecology and Evolution

539 Recombination: the process by which genomic regions are exchanged and broken up,

540 producing new combinations of alleles at different loci. Recombination occurs during meiosis

541 in eukaryotic cells.

542

543

544 


\section{Box 1. Pre-Genomic Era Criteria for Identifying Sympatric Speciation}

In their classic review of speciation, Coyne \& Orr [4] proposed four criteria that would need to be met in order for compelling case studies of sympatric speciation to be established. Given the restrictive conditions under which sympatric speciation is theoretically possible, these criteria for assessing empirical examples are equally stringent. Following, the argument of Mayr [8], they place the burden of proof on sympatric speciation and assume allopatric speciation as the null hypothesis. The four criteria can arguably be split in to two components, one specifying the biogeographic conditions, and the other component specifying the genetic criteria under which an empirical case study would make a compelling example of sympatric speciation (Figure I).

\section{Biogeographic Component}

1. Species must have largely or completely over-lapping geographic range (Figure IA).

2. The biogeographic and evolutionary history of the groups must make the existence of an allopatric phase very unlikely (Figure IB).

\section{Genetic Component}

3. Speciation must show substantial reproductive isolation (Figure IC).

4. Sympatric species must be endemic sister species or an endemic monophyletic species flock (Figure ID).

As with most aspects of the study of sympatric speciation, these criteria have been a point of contention. See Bolnick and Fitzpatrick [7] for an in-depth discussion and review of these conditions.

(A)

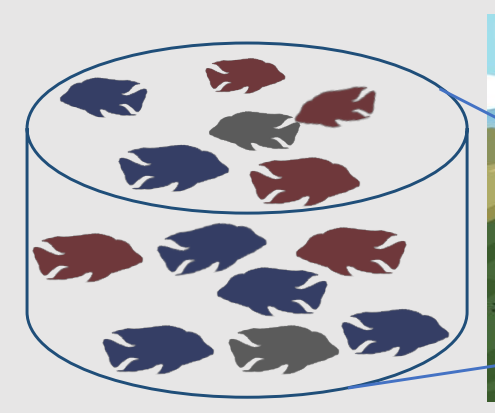

(B)

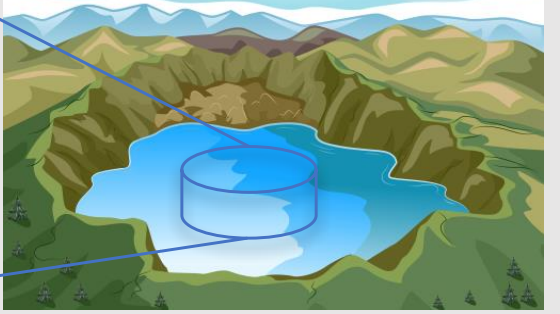

(C)

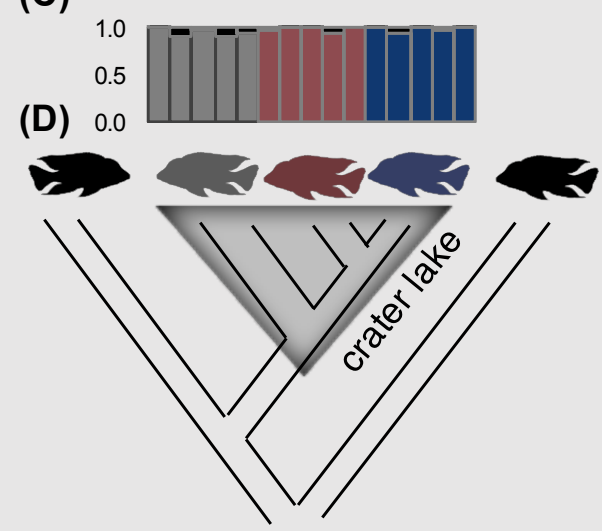

Figure I. Biogeographic and Genetic Criteria for Sympatric Speciation. Empirical case studies on crater lake cichlids were among the first to be considered as compelling examples of primary sympatric speciation [16-18]. (A) Cichlid species in these studies had distributions that overlapped and different species were in 'cruising range' sensu Mayr [7]. (8) The high rim of the caldera of these craters isolates the lake from neighbouring rivers, and the conical shape of the lake bottom prevents separate basins forming during periods of low water-level [16]. Thus, there are no geographical barriers to gene flow within the crater lake. (C) Analyses of nuclear DNA markers suggest that gene flow occurs predominantly within rather than between species (illustrated here with an admixture plot) [18]. (D) Phylogenetic analyses show that cichlid species within each lake form a monophyletic clade with respect to outgroups from neighbouring river systems, suggesting that they radiated in situ from a single shared ancestral population [16-18]. 


\section{Opinion Submission for Trends in Ecology and Evolution}

547 Figure 1 Evolutionary histories that could result in a monophyletic relationship among

548 sympatric sister species. Schematic tree figures (top) are coloured to indicate changes in

549 allele frequencies during divergence and introgression (indicated by horizontal arrows).

550 Schematic ancestry palettes (bottom) are coloured to indicate the differences in ancestry

551 proportions shared between the sympatric sister species and outgroups under each scenario.

552 (A) Speciation follows a single colonisation of an isolated 'island' habitat and divergence

553 during sympatry. Under this scenario, the three sympatric sister species would share a similar

554 proportion of their ancestry with outgroups. (B) Colonisation of an isolated 'island' habitat is

555 preceded by admixture with the outgroups followed by a period of panmixia could also result

556 in the three sympatric sister species sharing a similar proportion of their ancestry with

557 outgroups; however, colonisation by a structured meta-population or hybrid swarm could result

558 in the amount of shared ancestry with outgroups differing among ecotypes. (C) Multiple

559 independent colonisations of an isolated 'island' habitat over time, and episodic admixture

560 upon secondary contact would result in the introgressed species sharing more of their ancestry

561 with the outgroups most closely related to the source population of this secondary colonisation.

562 These three examples are not meant to be exhaustive, but simply illustrative of how different

563 evolutionary histories can result in the same majority-rule topology if evolutionary history is

564 modelled as a single bifurcating tree. This figure is adapted from reference [14]. 


\section{Opinion Submission for Trends in Ecology and Evolution}

567 Figure 2 Palaeogenomic sampling of divergent speciating lineages from sediment 568 cores. (A) An isolated lake is founded by a single lineage (grey). During a period of spatial 569 separation within the lake, two daughter lineages are derived (red and blue) and are adapted to 570 local ecological conditions and associated mate choice. Upon secondary contact, mate choice 571 maintains this segregation of the two lineages. Sampling the contemporary lineages from the 572 lake, one would reconstruct an ancestral history similar to that portrayed in Figure 1A, and

573 would be unable to distinguish whether reproductive isolation had become established despite 574 lineages having remained spatially overlapped throughout their post-colonisation history, or, 575 as in this case, whether reproductive isolation had developed during a period of spatial 576 isolation. (B) Sampling sediment cores of lakes and sequencing the sediment layers, or hard 577 body parts within them, provides a time series of genomic data that can elucidate the temporal 578 patterns of spatial overlap, in addition to the chronology and tempo of genomic changes 579 associated with adaptation and speciation, i.e. the onset of selection.

580 In the example shown, the sediment core has been drilled in the area used exclusively by the blue $581 \quad$ lineage during the allopatric phase. Sampling multiple cores would establish the approximate distribution of both lineages through space and time. 


\section{Opinion Submission for Trends in Ecology and Evolution}

582 Figure 3 Patterns of genomic differentiation due to sympatric and allopatric 583 divergence. (A) Schematic tree figures (top) are coloured to indicate changes in allele 584 frequencies at a large effect barrier locus during divergence and introgression (indicated by red 585 horizontal arrow). During divergence-with-gene-flow in sympatry, there is genome-wide 586 homogenisation due to ongoing gene-flow (indicated by black horizontal arrows). The 587 segregation of alleles in different incipient species at large effect barrier loci associated with 588 ecological adaptation and reproductive isolation will predate the mean genome-wide coalescent 589 time. This should be true whether the segregating alleles in barrier loci result from de novo 590 mutations (indicated by red star) during sympatry, standing variation that was present prior to 591 the sympatric phase, including from balanced polymorphisms, introgression and secondary 592 contact. Thus, such loci should stand out against a background of homogenised loci in genome593 wide scans. (B) In many scenarios where genome-wide incompatibilities have evolved during 594 allopatry, which preclude gene-flow upon secondary contact, then TMRCA of alleles at 595 incompatibility loci will fall within the range of the genome-wide mean TMRCA, and both 596 will predate secondary contact. This may not be ubiquitous. For example, balanced 597 polymorphisms which segregated upon speciation would still have a TMRCA that predated the 598 genome-wide mean. 

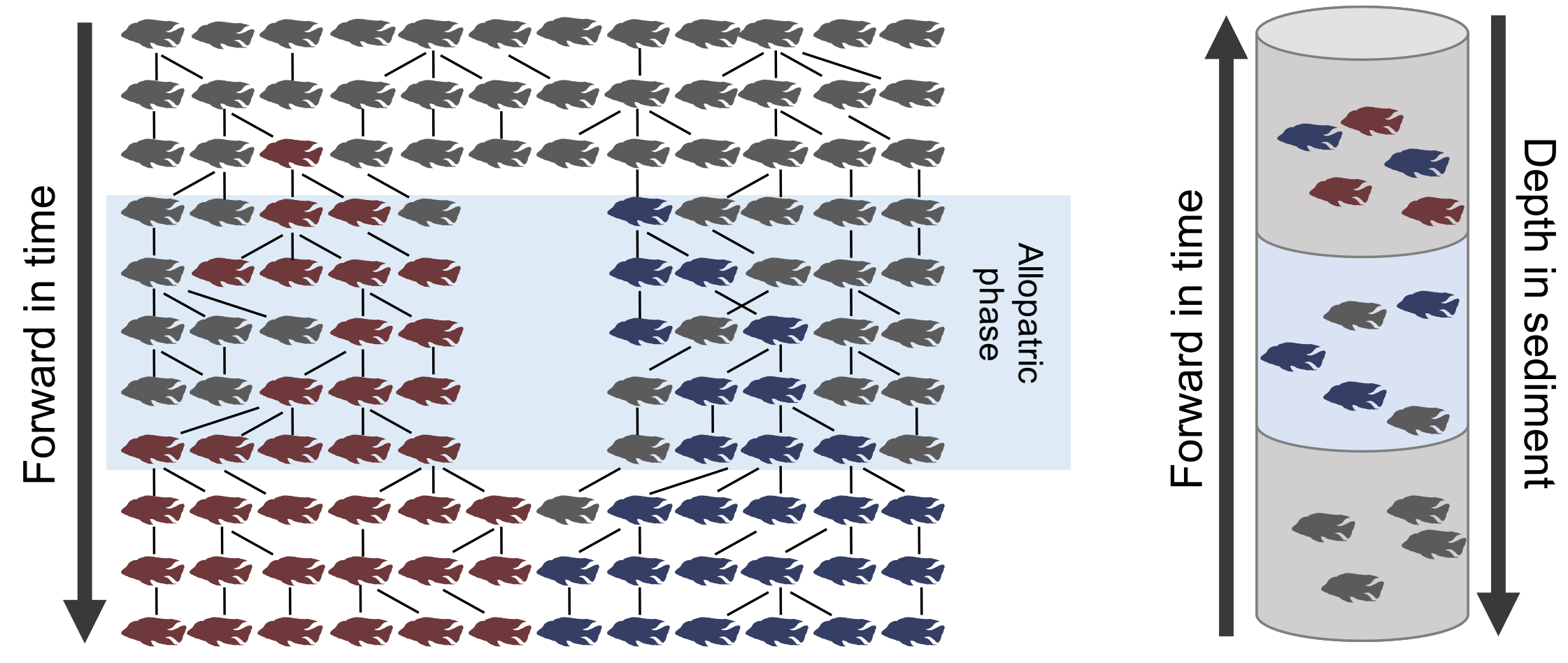
Figure $(\AA)$

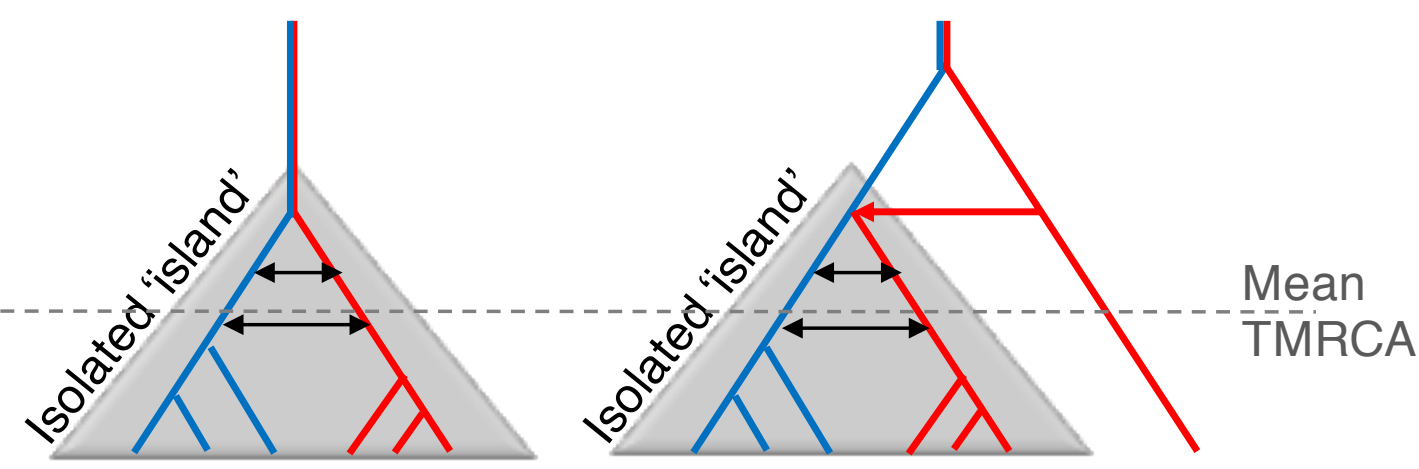
sympatric sister taxa sympatric sister taxa sympatric sister taxa outgroup

(B)

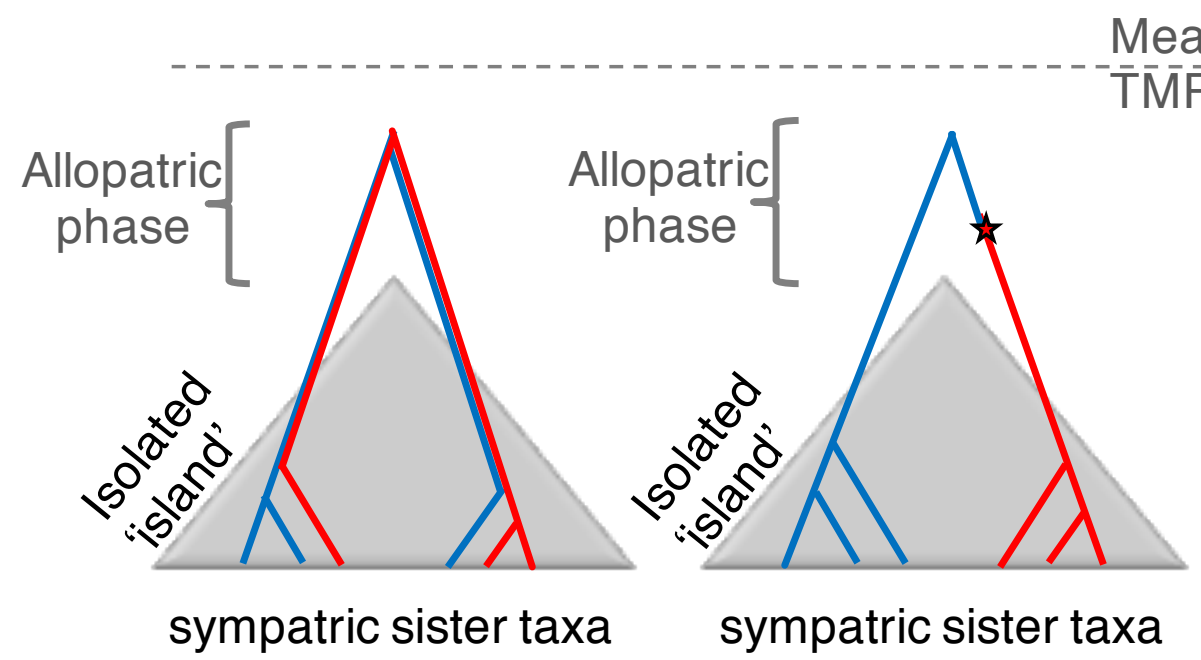

